\title{
$\mathrm{M}|\mathrm{R}| \mathrm{S}$ Internet Journal Nitride Semiconductor Research
}

\section{New buffer sublayers with crystal structure of cubic syngony for growing the heteroepitaxial films of nitride compounds of type $\mathbf{A}^{\mathrm{III}} \mathbf{B}^{\mathrm{V}}$ on sapphire substrates}

\author{
M.J. Kotelyanskii ${ }^{1}$, I.M. Kotelyanskii ${ }^{2}$ and V.B. Kravchenko ${ }^{2}$ \\ ${ }^{1} 312$ Quaker Church Road, Apt. D4, Flanders, NJ, 07869, \\ ${ }^{2}$ Institute of Radioengineering and Electronics, \\ (Received Thursday, May 6, 1999; accepted Thursday, October 28, 1999)
}

We propose the use of a buffer sublayer made of materials with crystal structure of cubic syngony to eliminate $30^{\circ}$ in-plane rotation of (0001) heteroepitaxial wurtzite type $\mathrm{A}^{\mathrm{III}} \mathrm{B}^{\mathrm{V}}$ nitride films with respect to the $(0001)$ or $(11 \overline{2} 0)$ working surface of the sapphire substrate. In these cases, the lattice parameter mismatch between the sapphire substrate surface and the semiconductor film is much smaller, and the cleavage planes of the sapphire and the semiconductor films with wurtzite structure forming the active region of a heterolaser are parallel. It is shown experimentally that using, for instance, $\mathrm{Nb}$ on (0001) $\mathrm{Al}_{2} \mathrm{O}_{3}$ or $\mathrm{NbN}$ on $(11 \overline{2} 0) \mathrm{Al}_{2} \mathrm{O}_{3}$, allows the elimination of the $30^{\circ}$ in-plane rotation of the (0001)AIN film with respect to the (0001) or (1150) working surface of the sapphire substrate .

\section{Introduction}

One of the major problems in development of semiconductor devices based on heteroepitaxial films of nitride compounds of type $\mathrm{A}^{\mathrm{III}} \mathrm{B}^{\mathrm{V}}$ is poor parameter reproducibility and fast degradation of the devices due to the high concentration of crystal structure defects in the films forming the active region. The high defect concentration in these films is due to the large lattice parameters mismatch between adjacent crystal planes of the substrate and of the semiconductor film. For monocrystalline substrates currently used for growing heteroepitaxial (0001) $\mathrm{GaN}$ films, the lattice mismatch is $16 \%$ for (0001) $\mathrm{Al}_{2} \mathrm{O}_{3}, 9.5 \%$ for $\mathrm{MgAl}_{2} \mathrm{O}_{4}$, and 3.5\% for SiC. A heteroepitaxial buffer sublayer of material with wurtzite crystal structure $(\mathrm{GaN}, \mathrm{AlN}, \mathrm{ZnO})$ is used to reduce the lattice parameter mismatch when the semiconductor layers are grown on (0001) $\mathrm{Al}_{2} \mathrm{O}_{3}$ surfaces [1]. This large mismatch in the heteroepitaxy of the hexagonal syngony materials with wurtzite structure on the (0001) sapphire surface is due to the $30^{\circ}$ in-plane rotation of the contacting (0001) plane of $\mathrm{Al}_{2} \mathrm{O}_{3}$ and the (0001) plane of wurtzite relative to each other. The reason for this rotation is that the most dense packing direction in the (0001) plane of $\mathrm{Al}_{2} \mathrm{O}_{3}$ is $\langle 1 \overline{1} 00\rangle \mathrm{Al}_{2} \mathrm{O}_{3}$, and in the wurtzite (0001) plane such direction is $\langle 1 \overline{1} 00\rangle$. Accord- ing to the criteria of mutual parallelism of the most densely packed directions in the contacting planes in heteroepitaxy, the direction $\langle 1 \overline{2} 10\rangle$ of $\mathrm{Al}_{2} \mathrm{O}_{3}$ is parallel to the wurtzite $\langle 1 \overline{2} 10\rangle$ direction. The translation period along $\langle 1 \overline{1} 00\rangle \mathrm{Al}_{2} \mathrm{O}_{3}$ is $2.747 \AA$, while for $\langle 1 \overline{2} 10\rangle \mathrm{GaN}$ it is $3.189 \AA$, leading to the large mismatch. Due to the large mismatch, the heteroepitaxial buffer sublayer of the material with wurtzite crystal structure can only grow on (0001) $\mathrm{Al}_{2} \mathrm{O}_{3}$ by the three-dimensional "island" growth mechanism. As it is known, this growth mechanism only allows for films with the "mosaic" monocrystalline structure, consisting of separate grains with large amount of grain boundaries and high defect concentration. In this article we propose a way to eliminate the $30^{\circ}$ in-plane rotation of the (0001) heteroepitaxial $\mathrm{A}^{\mathrm{III}} \mathrm{B}^{\mathrm{V}}$ nitride film with respect to the (0001) or $(11 \overline{2} 0)$ working surface of sapphire substrate.

\section{Results and Discussion}

We noticed that once the $30^{\circ}$ in-plane rotation of the (0001) heteroepitaxial nitride film with respect to the substrate is eliminated, the lattice parameter mismatch becomes substantially smaller. Indeed, two translations periods along $\langle 1 \overline{1} 00\rangle$ of $\mathrm{Al}_{2} \mathrm{O}_{3}(2 * 2.747=5.494 \AA$ ) coincides with the $0.5 \%$ accuracy with the a single 
translation along $\langle 1 \overline{1} 00\rangle \mathrm{GaN}$, equal to $5.52 \AA$, and two translations along $\langle 11 \overline{2} 0\rangle \mathrm{Al}_{2} \mathrm{O}_{3}$, equals $2 * 4.76=9.52$ $\AA$, this also coincides with three translations along $\langle 1 \overline{2} 10\rangle \mathrm{GaN}, 3 * 3.189=9.56 \AA$ with $0.5 \%$ accuracy. To eliminate this $30^{\circ}$ in-plane rotation of the film, we propose [2] to use a buffer sublayer of a material with cubic syngony, which for heteroepitaxy on (0001) or (1120) $\mathrm{Al}_{2} \mathrm{O}_{3}$ has the surface orientation $\{111\}$, and the direction $\langle 112\rangle$ in this plane is parallel to the $\langle 1 \overline{1} 00\rangle$ type direction of the $\mathrm{Al}_{2} \mathrm{O}_{3}$, lying in the working surface of the substrate. When a wurtzite type material is further grown on the $\{111\}$ surface of the cubic syngony material, the following epitaxial relationships hold:

1. $\{111\}$ planes of the cubic lattice and (0001) planes of wurtzite are parallel;

2 . $\langle 112\rangle$ directions of the cubic lattice and $\langle 1 \overline{1} 00\rangle$ directions of wurtzite are parallel.

Thus, if materials with cubic syngony are used for the buffer sublayer, the following orientation relationships can be obtained:

a) (0001) $\mathrm{Al}_{2} \mathrm{O}_{3} \|\{111\}$ cubic $\|(0001)$ wurtzite,

b) (112̄0) $\mathrm{Al}_{2} \mathrm{O}_{3} \|$ |111\}cubic \| (0001)wurtzite

in both cases: $\langle 1 \overline{1} 00\rangle \mathrm{Al}_{2} \mathrm{O}_{3} \|\langle 112\rangle$ cubic $\|$ $\langle 1 \overline{1} 00\rangle_{\text {wurtzite. }}$

Thus, if the buffer sublayer is prepared as we propose from material with crystalline structure of cubic syngony, with the lattice unit cell parameter $a$ such that the translation length along the $\langle 112\rangle$ direction of this lattice is very close to a multiple of the translation lengths along the $\langle 1 \overline{1} 00\rangle$ directions in the semiconductor film and in the substrate, an almost perfect lattice parameter match can be achieved, for instance, between (0001) $\mathrm{Al}_{2} \mathrm{O}_{3}$ and $\{111\}$ of the buffer layer on one side, and $\{111\}$ of the buffer sublayer and (0001) of the film with wurtzite structure, on the other side.

The cubic lattice unit cell parameter of the buffer sublayer material $a$ can then be determined from the relationship $a^{*} 2^{0.5} 3^{0.5}=\mathrm{n} * 2.747 \AA$, where $2.747 \AA$ is the translation period along $\langle 1 \overline{1} 00\rangle \mathrm{Al}_{2} \mathrm{O}_{3}$, and $n$ is an integer; this gives $a=1.125 n \AA$. The unit cell lattice parameters of the $\mathrm{Ga}$ and $\mathrm{Al}$ nitrides, as well as solid solutions of $\mathrm{Ga}$, In, and $\mathrm{Al}$, used in semiconductor devices, are within the range from 3.11 to $3.25 \AA$. Materials with a unit cell parameter $a$ less than $2.5 \AA$, or greater, than $13 \AA$ are practically absent. A reasonable choice would be to use as buffer sublayers materials with cubic syngony with the value of $a \sim 1.125 n \AA$, where $n$ equals $3,4,6$ or 8 . Values of 5,7 , or 9 are not acceptable for $n$, as in these cases translations along $\langle 112\rangle$ and $\langle 110\rangle$ of the (111) buffer layer plane, are not multiples of the translations along $\langle 1 \overline{1} 00\rangle$ and $\langle 11 \overline{2} 0\rangle$ of the semiconductor film. These values do not allow achievement of the desired result. Notice that in the case of heteroepitaxial deposition of semiconductor nitride film with the zinc blende polytype structure, the material of the heteroepitaxial buffer sublayer should also be chosen according to the conditions described above. Heteroepitaxial buffer sublayers can be implemented using material with cubic syngony with the crystal lattices of various types, like for instance $\alpha-\mathrm{Fe}, \mathrm{NaCl}$, and spinel. Sublayer materials can be dielectrics such as $\mathrm{BaCeO}_{3}, \mathrm{MgO}$, and $\mathrm{BP}$, conducting materials such as nitrides and carbides of niobium, hafnium, scandium, titanium and their solid solutions, or metals like niobium and tantalum. Buffer sublayers made of conducting material, can in addition serve as one of the electrodes of a semiconductor device. Heteroepitaxial layer structures may contain several buffer sublayers.

Our experiments show that using a $\{111\}$ niobium heteroepitaxial buffer sublayer allows the elimination of the $30^{\circ}$ in-plane rotation of $(0001) \mathrm{Al}_{2} \mathrm{O}_{3}$ with respect to the (0001) AlN film. The lattice parameter misfits along the mutually parallel $\langle 1 \overline{1} 00\rangle \mathrm{Al}_{2} \mathrm{O}_{3} \|\langle 112\rangle \mathrm{Nb}$ and $\langle 11 \overline{2} 0\rangle \mathrm{Al}_{2} \mathrm{O}_{3} \|\langle 110\rangle \mathrm{Nb}$ directions are less than $2 \%$. A very thin film of niobium nitride formed subsequently on the surface of the niobium buffer sublayer and the (0001) aluminum nitride film grown on it has practically coinciding (within $0,1 \%$ accuracy) atomic plane lattices.

The $30^{\circ}$ in-plane rotation between $\langle 1 \overline{1} 00\rangle \mathrm{Al}_{2} \mathrm{O}_{3}$ and $\langle 1 \overline{1} 00\rangle$ AlN for $(11 \overline{2} 0)$ sapphire substrates can also be removed by using $\{111\}$ niobium nitride barrier layer. The lattice parameter misfit is less than $2 \%$ along the mutually parallel $\langle 1 \overline{1} 00\rangle \mathrm{Al}_{2} \mathrm{O}_{3}$ and $\langle 112\rangle \mathrm{NbN}$ directions, and (0001) AlN films grown on it have practically coinciding atomic plane lattices.

Heteroeptaxial $\mathrm{Nb}, \mathrm{NbN}$ and AlN thin films on $(0001)$ and $(11 \overline{2} 0)$ sapphire substrates were deposited by the RF- magnetron sputtering method. The orientations of the films relative to the substrate was investigated by $X$-ray diffraction analysis; standard $\Theta-2 \Theta$ diffractometry and $\varphi-$ scans for pole figure drawing. We used a double-crystal diffractometer DRON-3 $\left(\mathrm{Cu}, \mathrm{K}_{\alpha 1}\right.$ X-ray tube). The XRD pattern of AlN grown on a $\mathrm{Nb}$ sublayer and on a (0001) $\mathrm{Al}_{2} \mathrm{O}_{3}$ substrate is presented in Figure 1. Figure 2 shows the representative $\mathrm{X}$-ray pole figures from AlN, $\mathrm{Nb}$ films and (0001) $\mathrm{Al}_{2} \mathrm{O}_{3}$ substrate. Figure 2 $(\mathrm{a}, \mathrm{b}, \mathrm{c})$ are pole figures taken for peaks from $\mathrm{AlN}$ $\{10 \overline{1} 1\}, \mathrm{Nb}-\{200\}$ and $\mathrm{Al}_{2} \mathrm{O}_{3^{-}}\{01 \overline{1} 2\}$, respectively. The results indicate that the in-plane orientations between (0001) AlN and (0001) $\mathrm{Al}_{2} \mathrm{O}_{3}$ changed when a 
$\mathrm{Nb}$ intermediate epitaxial layer is formed on the substrate. The appearance of six spots in Figure 2(b) for $\mathrm{Nb}\{200\}$ planes can be explained by the occurrence of the double positioning of the $\mathrm{Nb}$ film.

The orientation relationships were found to be the following:

$(0001)\langle 1 \overline{1} 00\rangle \mathrm{Al}_{2} \mathrm{O}_{3}||\{111\}\langle 112\rangle \mathrm{Nb} \|(0001)\langle 1 \overline{1} 00\rangle \mathrm{AIN} ;$

and

$(11 \overline{2} 0)[1 \overline{1} 00] \mathrm{Al}_{2} \mathrm{O}_{3}\|\{111\}[112] \mathrm{NbN}\|(0001)[1 \overline{1} 00] \mathrm{AIN}$

Notice, that the orientation relationships we obtained between the heteroepitaxial $\{111\} \mathrm{Nb}$ layer on (0001) $\mathrm{Al}_{2} \mathrm{O}_{3}$ and $\{111\} \mathrm{NbN}$ on (1120) $\mathrm{Al}_{2} \mathrm{O}_{3}$ are in agreement with earlier published experimental results presented in [3] and [4] correspondingly.

\section{Summary}

We propose use of a buffer sublayer of a material with cubic syngony crystal structure which has $\{111\}$ orientation for epitaxy on sapphire substrates and contains a direction of type $\langle 112\rangle$ parallel to the direction $\langle 1 \overline{1} 00\rangle$ in the surface plane of the sapphire substrate to eliminate the $30^{\circ}$ in-plane rotation of (0001) heteroepitaxial wurtzite -type $\mathrm{A}^{\mathrm{III}} \mathrm{B}^{\mathrm{V}}$-nitride films with respect to (0001) or $(1 \overline{1} \overline{2} 0)$ working surface of sapphire substrate. Implementation of this buffer sublayer allows:

1. acheivement of practically perfect plane mesh parameter match of adjacent planes, for instance, (0001) of the substrate and $\{111\}$ of the buffer sublayer on one side, and $\{111\}$ of the buffer sublayer and (0001) of the semiconductor film with wurtzite structure (for example, GaN), or $\{111\}$ for the cubic polytype zinc blende structure of the same film, on the other side.

2. elimination of the $30^{\circ}$ in-plane rotation between the cleavage planes of the sapphire substrate and of the wurtzite semiconductor layers. This is important for fabrication of heterolasers, as it contains Fabri-Perot resonators formed by the mirrors of heteroepitaxial semiconductor film cleavage planes.

It still remains to be verified experimentally that elimination of the $30^{\circ}$ in-plane rotation does indeed lead to better film quality.

\section{REFERENCES}

[1] H. Morkoc, S. Strite, G. B. Gao, M. E. Lin, B. Sverdlov, M. Burns , J. Appl. Phys. 76, 1363-1398 (1994).

[2] M.I.Kotelyanskii, I.M.Kotelyanskii, and V.B.Kravchenko "Semiconductor device based on heteroepitaxial $\mathrm{Ga}_{1-\mathrm{x}} \mathrm{Al}_{\mathrm{x}} \mathrm{N}$ films". Patent Application PCT/RU 98/00397 from 25.11.1998

[3] K. Masek, V. Matolin, Thin Sol. Films 317, 183 (1998).

[4] V. L. Noskov, Yu. V. Titenko, F. I. Korzhinsky, V. A. Komashko, Sov. Phys. Cryst. 25, 878 (1980).

\section{FIGURES}

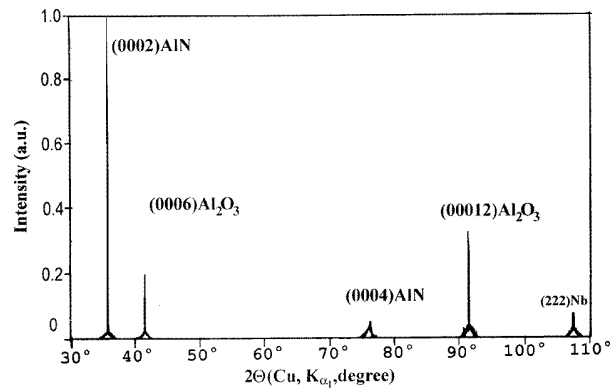

Figure 1. XRD $(\Theta-2 \Theta)$ scan of AlN film, grown on $\mathrm{Nb}$ sublayer and (0001) $\mathrm{Al}_{2} \mathrm{O}_{3}$ substrate.

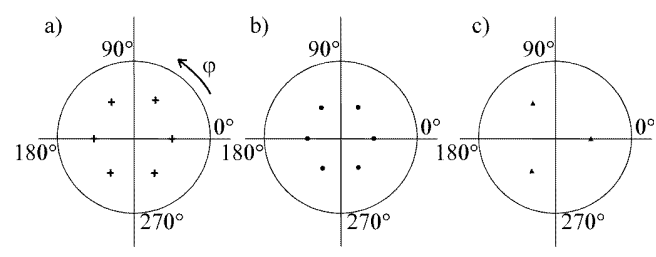

$$
\begin{aligned}
& \text { Fig.2 Representive X-ray pole figures from: } \\
& \text { a) (0001) AlN film (+) - peaks }\{10-11\} \text {; } \\
& \text { b) (111) } \mathrm{Nb} \text { film }(\bullet) \text { - peaks }\{200\} \text {; } \\
& \text { c) }(0001) \mathrm{Al}_{2} \mathrm{O}_{3} \text { substrate (.) - peaks }\{01-12\}
\end{aligned}
$$

Figure 2. Representative X-ray pole figures from: a) (0001) AlN film (pluses) - peaks $\{10 \overline{1} 1\}$; b) (111) Nb film (filled circles) peaks $\{200\}$ c) $(0001) \mathrm{Al}_{2} \mathrm{O}_{3}$ substrate (triangles) peaks $\{0 \overline{1} 12\}$ 